\title{
SMMiL-E, Japanese Bio-MEMS Laboratory in France, and the Role of Scientific Direction
}

\author{
Teru Okitsu, ${ }^{1,4^{*}}$ Eric Leblanc, ${ }^{2,4}$ Dominique Collard, ${ }^{1,3,4}$ and Hiroyuki Fujita ${ }^{1,4}$ \\ ${ }^{1}$ Institute of Industrial Science, University of Tokyo, 4-6-1 Komaba, Meguro-ku, Tokyo 153-8505, Japan \\ ${ }^{2}$ Department of Oncological Gynecological Surgery, Centre Oscar Lambret, \\ 3 Rue Frédéric Combemale, 59000 Lille, France \\ ${ }^{3}$ LIMMS/CNRS-IIS UMI 2820, Lille-Site, Institut pour la Recherche sur le Cancer de Lille, \\ Boulevard du Pr Jules Leclercq, 59000 Lille, France \\ ${ }^{4} \mathrm{CNRS} / \mathrm{IIS} / \mathrm{COL} /$ Lille University SMMiL-E Project, CNRS Délégation Nord-Pas de Calais et Picardie, \\ 2 rue de Canonniers, Lille, Cedex 59046, France
}

(Received April 25, 2019; accepted July 16, 2019)

Keywords: SMMiL-E, bio-MEMS, cancer medicine, translational research, scientific direction

The first overseas laboratory of the Institute of Industrial Science (IIS), the University of Tokyo, SMMiL-E, Seeding Microsystems in Medicine in Lille-European Japanese Technologies against Cancer-, has been established in Lille, France. There, the microsystems developed in LIMMS, Laboratory for Integrated Micro Mechatronic Systems, a FrenchJapanese laboratory at the IIS are applied to cancer therapies through interdisciplinary and international collaborations. A new function, the "Scientific Direction", has been implemented to ensure that these collaborations are effective and productive. One French and one Japanese medical doctor supervise and guide the research within SMMiL-E in their role as Scientific Directors. With the continuing growth of SMMiL-E, the Scientific Direction must also expand to uphold the quality of the collaborative projects. An efficient Scientific Direction is a key to the success of the SMMiL-E endeavour.

\section{SMMiL-E}

SMMiL-E, Seeding Microsystems in Medicine in Lille-European Japanese Technologies against Cancer-, has been launched as a French-Japanese-collaborative research program, and the platform for SMMiL-E was inaugurated in Lille, North France, in June 2014 as the first laboratory of the Institute of Industrial Science (IIS), The University of Tokyo, located overseas. The mission of SMMiL-E is to implement bio-MEMS technologies developed in Japan into cancer medicine in France. These bio-MEMS technologies are developed in LIMMS, Laboratory for Integrated Micro Mechatronic Systems, where the researchers from France and their host professors at the IIS are working on microsystems. Thus, SMMiL-E is recognized as the mirror structure of LIMMS, and it has been named to include this recognition (Fig. 1). ${ }^{(1-3)}$

SMMiL-E has three goals in its research against cancer: (1) to develop clinical bio-MEMS

*Corresponding author: e-mail: okitsu@iiis.u-tokyo.ac.jp https://doi.org/10.18494/SAM.2019.2375

This is an updated version of an article that appeared in Seisan-kenkyu 70 (2018) 189 (in Japanese). 


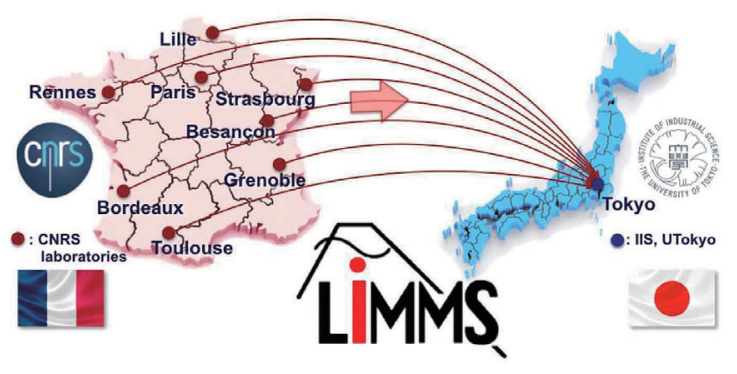

(a)

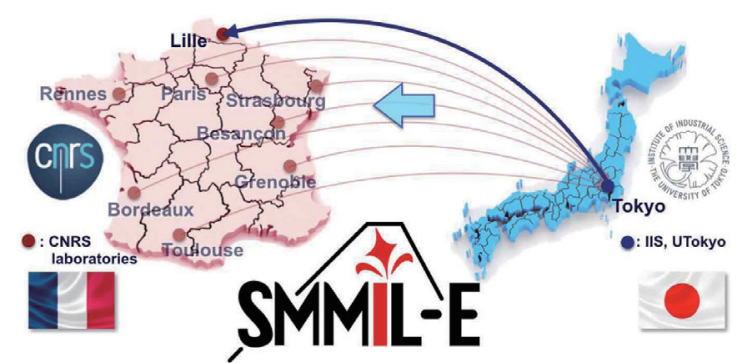

(b)

Fig. 1. (Color) (a) LIMMS and (b) SMMiL-E.

devices for cancer medicine, (2) to discover new drugs and phenomena related to cancer research and therapy, and (3) to establish new clinical protocols based on these discoveries. Within the SMMiL-E framework, engineers, biologists, and clinicians collaborate to achieve these goals with work spanning from basic research to preclinical testing. Throughout this research cycle, biologists and clinicians provide advice and clinical samples, while engineers develop new bio-MEMS devices and technologies that will be useful in oncological biology (Fig. 2).

As clearly shown in the development flow in SMMiL-E (Fig. 2), the pivotal activity is for engineers to develop their bio-MEMS technologies and to implement them in cancer diagnosis and therapies. Therefore, the research activities in SMMiL-E are classified as being "seedoriented" rather than "need-oriented". During the course of technological development, biologists and clinicians support engineers while maintaining not only positive mental attitudes for building up the technologies together with engineers but also expectations that the final outcomes will solve the issues of their interest in the near future. This specific research framework of SMMiL-E is based on the trust of French biologists and clinicians in the performance of the engineers of IIS, and this trust has become relevant because of the innovative results that LIMMS at the IIS has been achieving for over twenty years.

\section{Research Activities in SMMiL-E}

The scientific activities of SMMiL-E encompass bio-MEMS research against cancer, including technological development and biology-related experiments. The aim of the projects is to bridge fundamental and clinical research around the following four work packages (WPs) related to scales, from nano/micro to macro, of biological targets: WP1, biomolecular mechanisms; WP2, cellular evaluation; WP3, cell-cell interactions; WP4, new tissue. By means of upstream research, this program targets a more effective method of disease detection and the improved efficacy of therapy and post-treatment monitoring to enable better care of patients. Six projects have been labeled (LP) in SMMiL-E under the four WPs and are currently being actively investigated.

LP1. Mechanical nanotweezers (SNT) and microfluidic setup for the direct assay of DNA damage by therapeutic radiation beams.

LP2. Circulating cancer cell (CTC) extraction and capture in microfluidic format. 


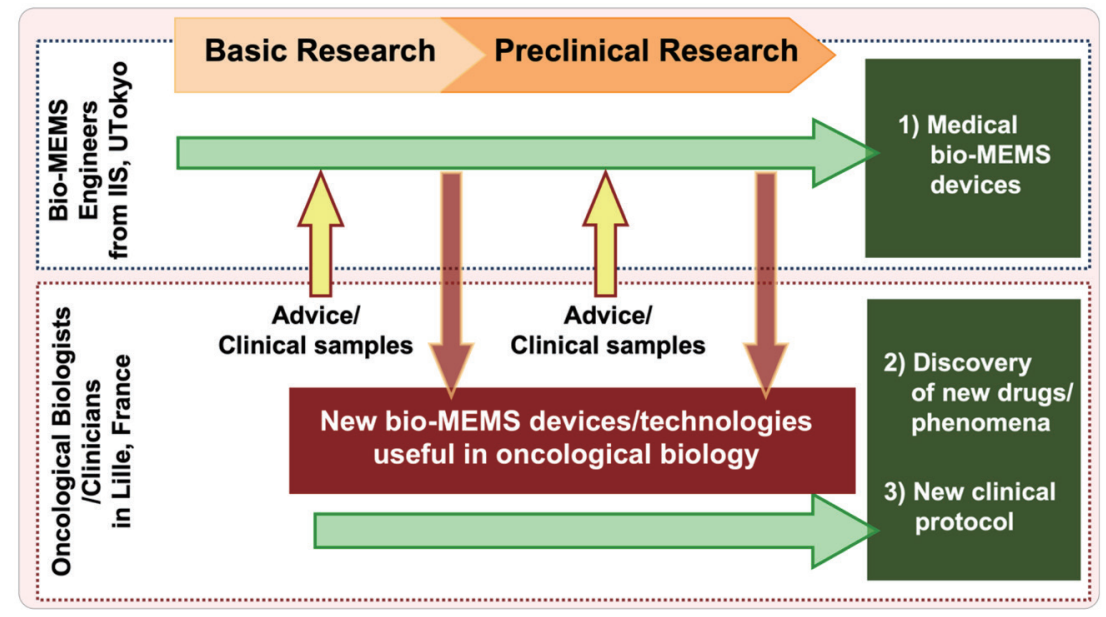

Fig. 2. (Color) Development flow in SMMiL-E.

LP3. Biomechanical monitoring of breast cancer stem cell (CSC) responses and reprogramming of non-CSCs to CSCs following irradiation or drug applications.

LP4. In vitro tumor angiogenesis and cellular motility.

LP5. Adhesion of pancreatic cancer cells in liver microvasculature in a microfluidic format for high-throughput screening with impedance spectroscopy sensing.

LP6. Autologous mesothelial cell-laden hydrogel sheet for prevention of postsurgical abdominal adhesions.

The following are the outlines of these projects,

In LP1 under WP1, the project team is developing a MEMS device that can monitor the degree of DNA degradation under X-ray irradiation in real time. This device directly traps a DNA bundle between the probes and the degradation is measured by reducing the rigidity of the bundle calculated from its resonance frequency response. ${ }^{(4)}$

In LP2 under WP2, the project team is developing a microfluidic device that can capture and extract circulating tumor cells (CTCs) from whole blood samples of cancer-carrying patients. This device is aimed toward increasing the accuracy of the diagnosis of cancer and monitoring the state of cancer. CTCs are captured and extracted by structural trapping and immunoaffinity binding. ${ }^{(5,6)}$ The microfluidic device is similar in size and shape to a commercially available 96-well plate, and consists of a polydimethylsiloxane (PDMS) microfluidic chip and a glass substrate. The microfilter is integrated in each well, and the inner surfaces of all the wells including the microfilters are coated with antibodies to bind CTCs. The pore of each microfilter is $7.5 \mu \mathrm{m}$ in height and $25 \mu \mathrm{m}$ in width (Fig. 3). Cancer cells spiked in blood were successfully captured in the device [Fig. 3(c)].

In LP3 under WP2, the project team is developing a device that can measure the mechanical characteristics of single cells in a high-throughput manner to elucidate the characteristic distribution of cancer cells. This device is being developed using a combination of MEMS technology and microflow control. ${ }^{(7)}$ The device contains a pair of opposing microscale tips 


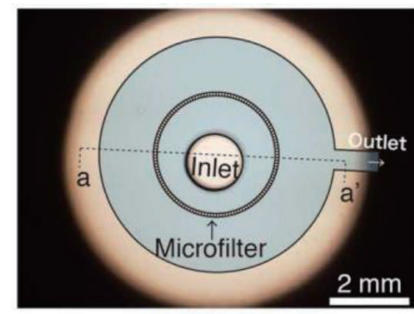

(a)

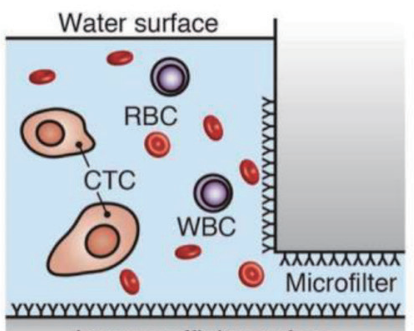

(d)

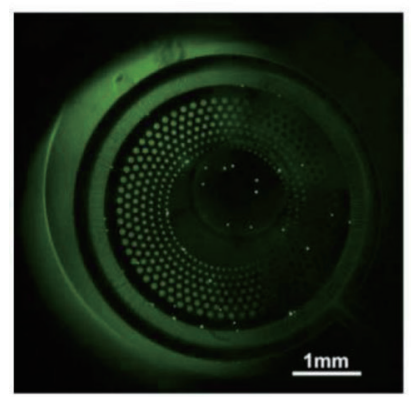

(g)

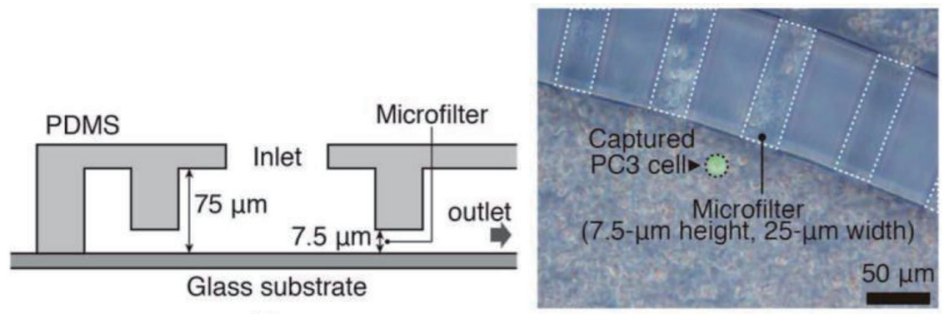

(b)

(c)

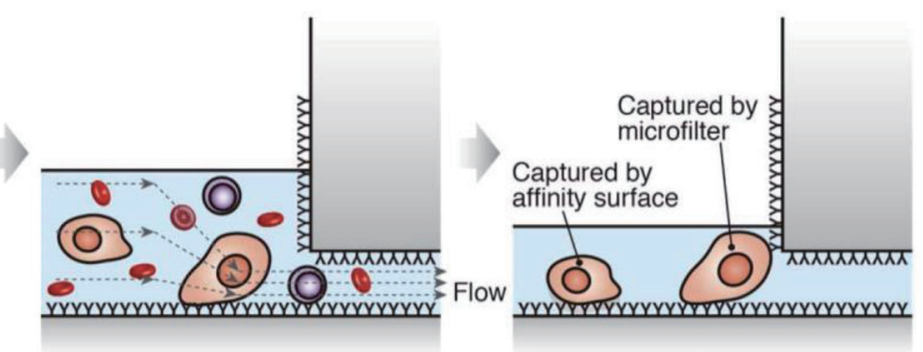

(e)

(f)

Fig. 3. (Color) Microfluidic device used in LP2 to capture and extract CTCs from whole blood samples of cancercarrying patients. (a) Developed CTC separation device. (b) Cross section along a-a' in Fig. 3(a). (c) PC3 cell (prostate cancer cell line) separated from a blood sample in the device. (d-f) Schematic illustration of how this device extracts CTCs through both cell-size-based and immunoaffinity-based cell separations. (g) Image of spike-in experiment with fluorescent-dye-stained cancer cell line. Parts (a)-(f) are from Jiro Kawada, Shohei Kaneda, Soo Hyeon Kim, and Teruo Fujii. Microfluidic Approach to Cell Handling and Measurement, in Intelligent Nanosystems for Energy, Information and Biological Technologies (Eds. J. Sone and S. Tsuji), 85-106, Copyrighted by Springer Nature (2016), used with permission from Springer Nature. ${ }^{(5)}$ Part (g) is from the SMMiL-E website. ${ }^{(6)}$

located in the microchannel. One of the tips actuates with a maximum displacement of 14 $\mu \mathrm{m}$ in order to compress cells that have flowed in the microchannel and have been captured between the tips. At the same time, the other tip vibrates with a small amplitude and senses the resonance frequency of the captured cells (Fig. 4).

In LP4 under WP3, the project team is developing a biochip integrated with both the reconstructed microvasculature and the cancer tissue. This device is aimed at screening anticancer drugs possessing the characteristics of antivascular agents. ${ }^{(8,9)}$

In LP5 under WP3, the project team is developing a bio-MEMS device including a biochip integrated with a reconstructed vascularized hepatic tissue to reproduce pancreatic cancer 


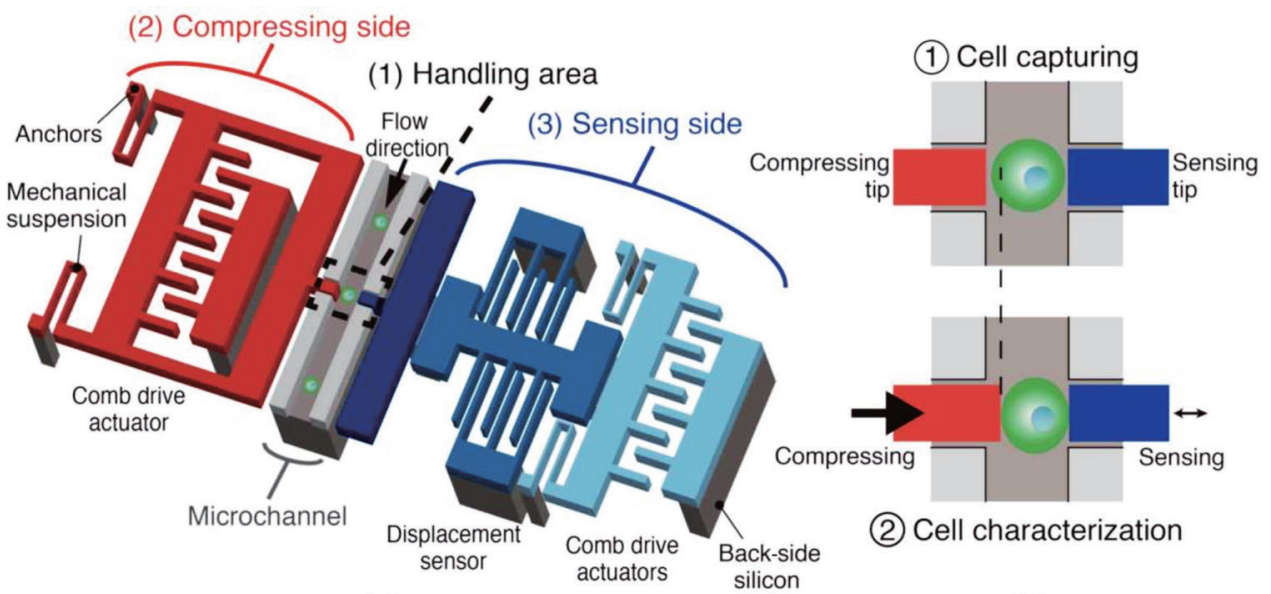

(a)

(b)

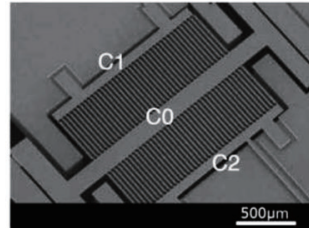

(c)

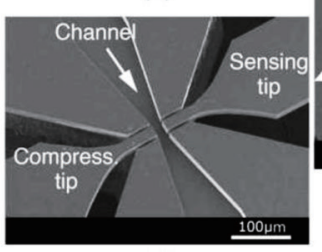

(d)

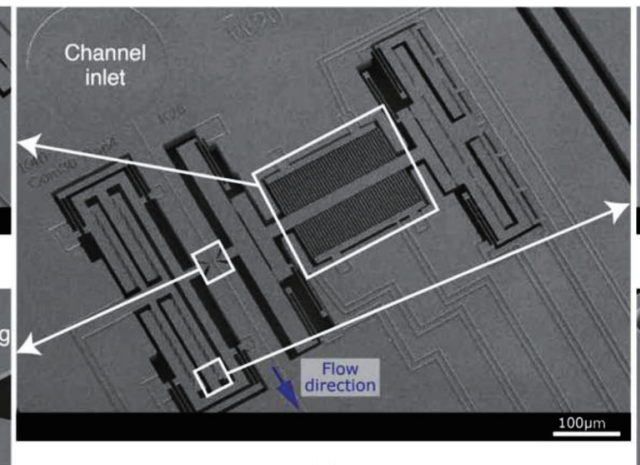

(e)

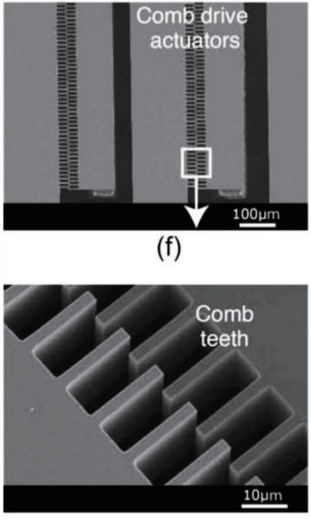

(g)

Fig. 4. (Color) Device used in LP3 to measure the mechanical characteristics of single cells in a highthroughput manner to elucidate the characteristic distribution of cancer cells. (a) Schematic image of the device configuration with a pair of opposing microscale tips in the microchannel. (b) Working principle of cell capture and characterization. (c-g) SEM images of the fabricated device: (c) displacement sensor based on differential capacitors, (d) opposing tips accessing the handling area, (e) an overview of the device, and (f) electrostatic comb drive actuators with (g) a close-up view. Parts (a)-(g) are from Yuki Takayama, Grégoire Perret, Momoko Kumemura, Manabu Ataka, Samuel Meignan, Stanislav L. Karsten, Hiroyuki Fujita, Dominique Collard, Chann Lagadec, and Mehmet Cagatay Tarhan. Developing a MEMS Device with Built-in Microfluidics for Biophysical Single Cell Characterization. Micromachines 9 (2018) 275. https://doi.org/10.3390/mi9060275. ${ }^{(7)}$

metastasis to the liver in vivo. This device is aimed at revealing the pathophysiology of pancreatic cancer metastasis to the liver and contributing to pancreatic cancer therapy. ${ }^{(10)}$

In LP6 under WP4, the project team is developing a regenerated peritoneal tissue using fibershaped collagens that serve as the scaffold for mesothelial cells. The fiber-shaped collagens are fabricated by applying the technology to control microflow. ${ }^{(11)}$ The team is aiming at rapid reperitonealization and at preventing peritoneal adhesion after surgery to remove advanced intraabdominal or intrapelvic cancer. ${ }^{(12)}$ 


\section{Structure of SMMiL-E and Scientific Direction}

The platform of SMMiL-E is located within the university-hospital site, to be nearest to medical teams in Lille, France. This is the first overseas laboratory of the IIS of The University of Tokyo, and this venture is backed by CNRS, Centre Oscar Lambret, and University of Lille, as a UMI; this is an International Join Unit, the mirror site of LIMMS/CNRS-IIS UMI-2820. In this platform, SMMiL-E allows Japanese engineers from the IIS of The University of Tokyo and French oncological biologists/clinicians to collaborate on the application of bio-MEMS to cancer research as well as cancer diagnosis and therapy [Fig. 5(a)]. Consequently, all the projects of SMMiL-E are not only international but also interdisciplinary collaborations, and many issues are very likely to arise. Since solving these issues is presumed to be difficult for the researchers or clinicians concerned, a new function called the "Scientific Direction" has been implemented to ensure that these collaborations proceed smoothly and fruitfully. Specifically, two medical doctors, one French and one Japanese, have been selected and assigned as Scientific Directors. These directors collaborate closely with each other and facilitate clear communication among the researchers and clinicians by overcoming possible issues that may arise as a result of the differences in, for example, the terminology used and how the members behave routinely in their own fields, and by preventing them from being estranged from each other [Fig. 5(b)].

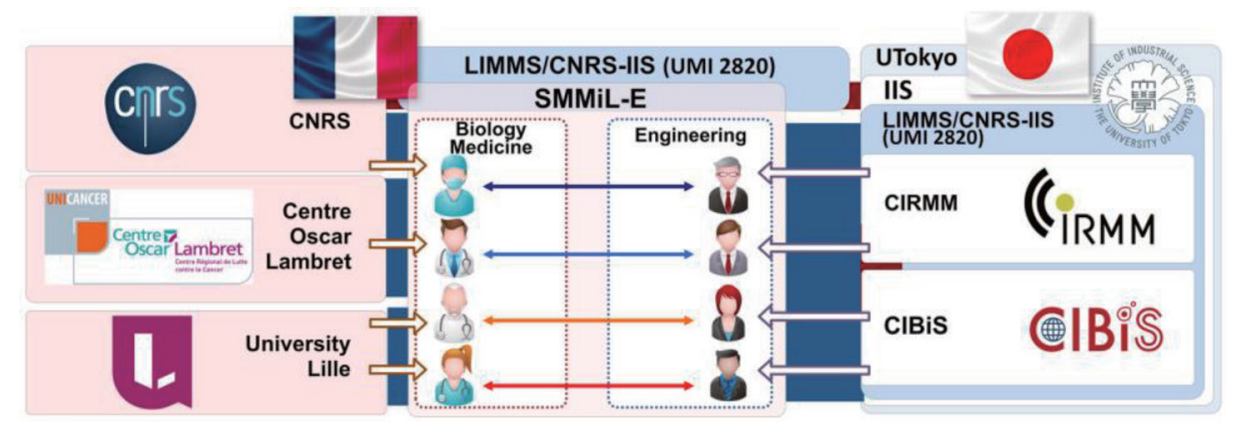

(a)

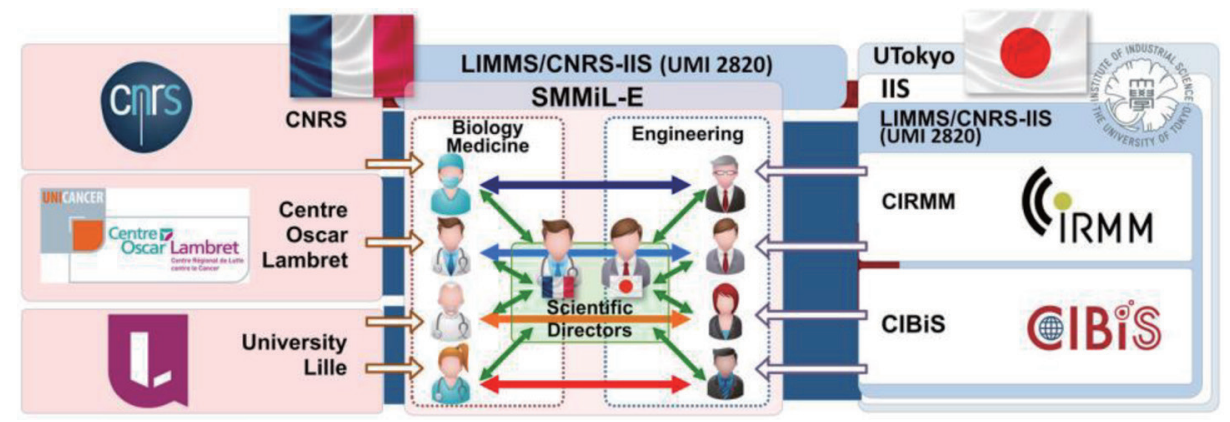

(b)

Fig. 5. (Color) (a) Structure of SMMiL-E. (b) Scientific Direction in SMMiL-E. 


\section{IBEC: LIMMS office in Lille (13) $^{(13)}$}

On July 2, 2015, one year after the signing of the SMMiL-E project agreement, the IBEC, IIS/UTokyo Bureau for European Collaboration, was opened in Lille, on the premises of the Regional Delegation of CNRS and Lille University. The function of this IBEC is to support the development of the SMMiL-E project as well as to extend the collaboration network in Europe. LIMMS has also opened an administrative office on the same site, providing a convenient secondary location in Lille in addition to its main location in Tokyo.

Lille is situated in northeastern France near the Belgian border and is centrally located within a dynamic and dense network of European cities and countries. Paris and its international airport, Brussels (Belgium) and the institutions of the European Union, and London (UK) are all easily accessible by train (Fig. 6). The tremendous advantages to placing the IBEC, IIS/UTokyo Office, in this dynamic eurometropolis near Europe's decision-making hub are undeniable for forging scientific partnerships with Europe. Lille also boasts an outstanding academic community that supports over 100000 students in universities, engineering, business

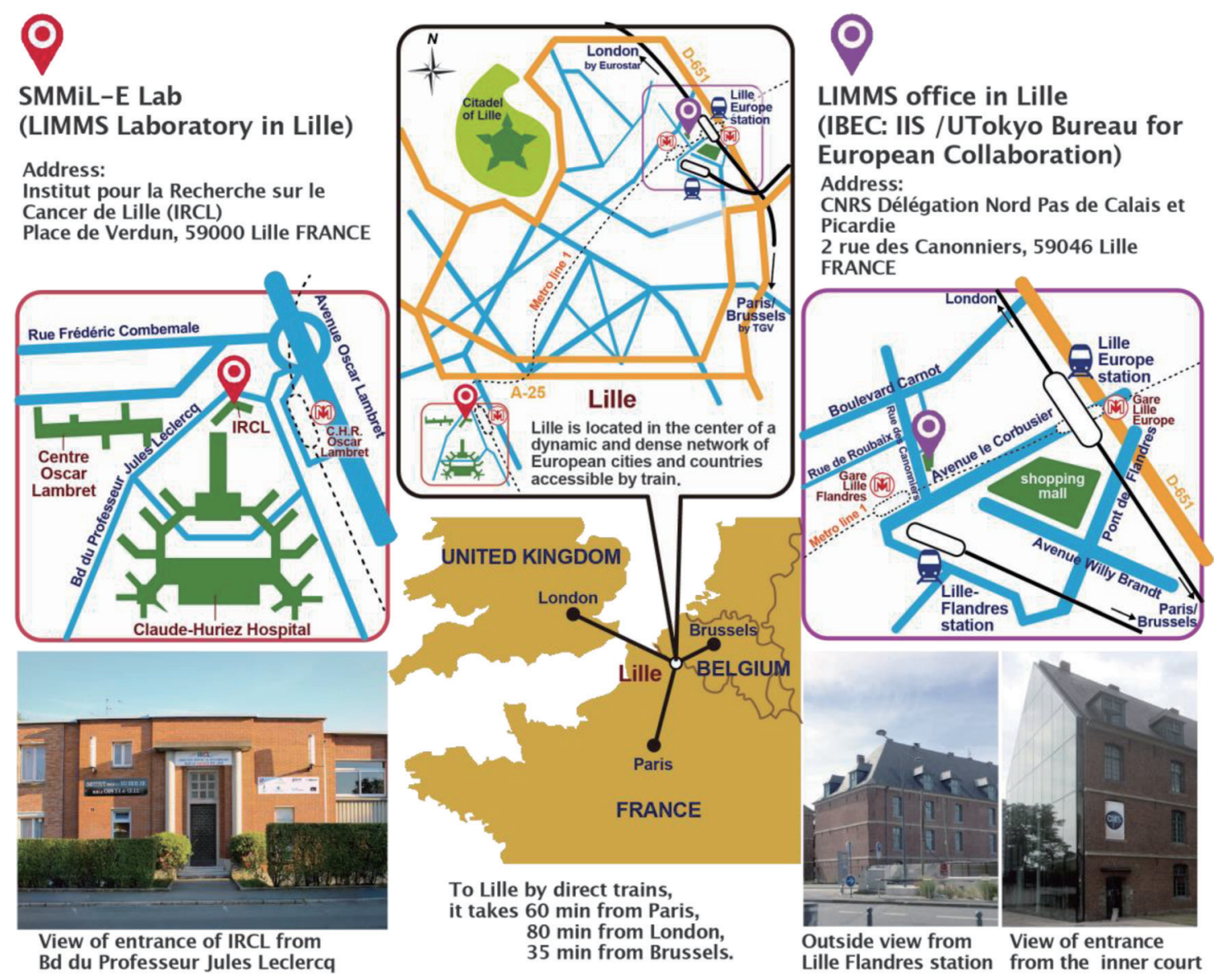

Fig. 6. (Color) Locations of SMMiL-E lab and IBEC in Europe. 
schools, and French best schools (Grandes Écoles) and provides a knowledge, research, and technological pool of excellence. The premier attraction, however, is that the largest topranking hospital-university campus in Europe ${ }^{(14)}$ comprising several specialized hospitals, schools, and research centers and offering state-of-the-art clinical medicine, is found in Lille. It is the ideal site for the IIS/UTokyo SMMIL-E program, which is dedicated to integrating bioMEMS research into the clinical research against cancer.

\section{Perspectives of SMMiL-E}

The inception of SMMiL-E can be traced back to an e-mail from France to Japan in December 2011. ${ }^{(15)}$ Two years and a half later, in June 2014, SMMiL-E was launched, when IIS/UTokyo, CNRS, University of Lille, Centre Oscar Lambret signed the Research and Collaboration Agreement for SMMiL-E in Lille. Thereafter, SMMiL-E has been performing bio-MEMS experiments supported by research grants, inaugurating a new cleanroom facility, and installing equipment and instruments (Table 1). Currently, there are six engineers from IIS/UTokyo who are staying in Lille and collaborating with the local oncology biologists/ clinicians: three Japanese, two French, and one Turkish. These three non-Japanese engineers have research experience in LIMMS at IIS/UTokyo and have succeeded in producing research results in SMMiL- $\mathrm{E}^{(4-10,12)}$ in cooperation with the professors belonging either to the Center

Table 1

Notable events before and after the launch of SMMiL-E .

2011

18 Dec Prof. Lartigau and Prof. Cleri proposed, through e-mail to Prof. Collard, the irradiation of DNA bundles held by SNT*

20 Dec Prof. Fujita agreed, at his lab meeting, to transfer the complete setup of SNT to Lille in France. 2012

7 Feb Upon the successful performance of SNT experiments in Lille, the Tweez'RT ${ }^{* *}$ project was created. 2013

Sep The Tweez'RT project was selected by the PhysiCancer plan. 2014

9 Dec A workshop in IIS, UTokyo, became the basis of SMMiL-E.

12 May SMMiL-E logo contest held and won by Dr. Laurent Jalabert and Ms. Stephanie Barbez. The logo is a mirror image of the original LIMMS logo, representing the parallel nature of these two organizations.

2014

16 June SMMiL-E agreement officially signed in Lille.

2015

2 July IBEC, "IIS/UTokyo Office for European Collaboration" opened in Lille.

10 July SMMiL-E support acquired as part of the CPER ${ }^{* * *}$ framework.

17 Dec Scientific Direction in SMMiL-E started by accepting Dr. Leblanc and Prof. Okitsu as the Scientific Directors.

2017

15 Dec SMMiL-E facilities at IRCL inaugurated in Lille.

2018

9 Nov Director General of IIS, Prof. Kishi, visited SMMiL-E in Lille.

3 Dec SMMiL-E Steering Committee held at Centre Oscar Lambret in Lille.

\footnotetext{
*SNT: silicon nanotweezers

** Tweez'RT: mechanical nanotweezers and microfluidic setup for the direct assay of DNA damage by therapeutic radiation beams

${ }^{* * *}$ CPER: French State-Region plan contract
} 
for International Research on Integrative Biomedical Systems (CIBiS) or to the Centre for Interdisciplinary Research on Micro-Nano Methods (CIRMM) of IIS/UTokyo (Fig. 5). These achievements in SMMiL-E have commanded considerable attention not only in Lille but also in northern France, and the activities of SMMiL-E have attracted much interest from oncology researchers there. As a result, a couple of local researchers expressed a strong desire to be exclusively engaged in SMMiL-E, and they successfully realized their desires in 2018. Moreover, several local research projects currently in the preliminary stages are in the wings as candidates for being labeled as new SMMiL-E projects. These circumstances will definitely lead to the expansion of the scale of SMMiL-E and the development of its research capabilities.

Accompanying the growth of SMMiL-E, the issue of how to maintain or even enhance the quality of the activity in SMMiL-E will probably arise. To cope with this issue, SMMiL-E is extending the function of the Scientific Direction. In addition to the conventional function of supporting the smooth and fruitful progress of the ongoing labeled projects, SMMiL-E expects the Scientific Direction to deal with the evaluation process for labeling new projects. SMMiL-E has imposed the following restrictions on the labeling of new projects, (1) First, the projects must have a project structure that includes a close connection with researchers working at IIS/ UTokyo, (2) Secondly, they must be clinically and scientifically attractive, must have important rationales, and must be significantly original. (3) Finally, they must have highly feasible goals. Furthermore, when the projects do not satisfy these conditions, the Scientific Direction is also expected to support their improvement. Specifically, the Scientific Directors evaluate the issues that dissatisfy the conditions and attempt to resolve these issues by introducing necessary collaborators to complete the proper project structure and to establish feasibility in achieving their research goals. Ultimately, SMMiL-E aims to facilitate the setting up and realization of a comprehensive research program for applying bio-MEMS against cancer in a sustainable international high-level collaboration between bio-MEMS engineers and oncology biologists/ clinicians. To attain this aim, it is crucial to efficiently fulfill the functions of the Scientific Direction that is unique to SMMiL-E.

\section{Acknowledgments}

This work was supported partially by the JSPS Core-to-Core Program, A. Advanced Research Networks: "Japan-Europe Research Hub for Translational Medical Engineering (JETMeE)". The authors acknowledge the support from SMMiL-E partners, the French StateRegion plan contract CPER, and IRCL (Research Institute against Cancer of Lille). The authors thank M. Kumemura (Kyushu Institute of Technology) for useful comments on the manuscript. The authors also thank T. Fujii (The University of Tokyo) and S. Kaneda (Kogakuin University) for providing original high-resolution images for Fig. 3, and M. C. Tarhan (LIMMS, SMMiL-E) for providing original high-resolution images for Fig. 4.

\section{References}

1 T. Okitsu, E. Leblanc, D. Collad, and H. Fujita: Seisan-kenkyu 70 (2018) 189 (in Japanese). https://doi. org/10.11188/seisankenkyu. 70.189 
2 http://smmil-e.com/

3 http://limmshp.iis.u-tokyo.ac.jp/

4 G. Perret, T. Lacornerie, F. Manca, S. Giordano, M. Kumemura, N. Lafitte, L. Jalabert, M. C. Tarhan, E. F. Lartigau, and F. Cleri: Microsyst. Nanoeng. 2 (2016) 16062.

5 J. Kawada, S. Kaneda, S. H. Kim, and T. Fujii: Intelligent Nanosystems for Energy, Information and Biological Technologies, J. Sone and S. Tsuji, Eds. (Springer, Tokyo, 2016) p. 85.

$6 \mathrm{http}: / / \mathrm{smmil}-\mathrm{e} . \mathrm{com} / \mathrm{research} /$

7 Y. Takayama, G. Perret, M. Kumemura, M. Ataka, S. Meignan, S. Karsten, H. Fujita, D. Collard, C. Lagadec, and M. Tarhan: Micromachines (Basel) 9 (2018) 275.

8 J. Pauty, R. Usuba, I. Cheng, L. Hespel, H. Takahashi, K. Kato, M. Kobayashi, H. Nakajima, E. Lee, F. Yger, F. Soncin, and Y. Matsunaga: EBioMedicine 27 (2018) 225.

9 R. Usuba, J. Pauty, F. Soncin, and Y. Matsunaga: Biomaterials 197 (2019) 305.

10 M. Danoy, M. Shinohara, A. Rizki-Safitri, D. Collard, V. Senez, and Y. Sakai: Integr. Biol. (Camb) 9 (2017) 350 .

11 H. Onoe and S. Takeuchi: Drug Discov. Today 20 (2015) 236.

12 L. Bresson, E. Leblanc, A. Lemaire, T. Okitsu, and F. Chai: Surgery 162 (2017) 863.

13 http://www.iis.u-tokyo.ac.jp/topics/IISNEWS/2016toku.pdf

14 http://www.chru-lille.fr/

15 N. Frances, B. J. Kim, H. Fujita, E. Lartigau, T. Fujii, and D. Collard: Seisan-kenkyu 67 (2015) 131. https://doi. org/10.11188/seisankenkyu.67.513 